\title{
A CLDN18.2-Targeting Bispecific T Cell Co-Stimulatory Activator for Cancer Immunotherapy
}

Jie Liang'

Huihui Zhang'

Yue Huang'

Lilv Fan'

Fanlin $\mathrm{Li}^{1}$

Min $\mathrm{Li}^{1}$

Yaping Yan'

Junshi Zhang'

Zeyu Li'

Xuanming Yang $\mathbb{D}^{1,2}$

'Sheng Yushou Center of Cell Biology and Immunology, School of Life Sciences and Biotechnology, Shanghai Jiao Tong University, Shanghai, 200240, People's Republic of China; ${ }^{2}$ Joint International Research Laboratory of Metabolic \& Developmental Sciences, Shanghai Jiao Tong University, Shanghai, 200240, People's Republic of China
Correspondence: Xuanming Yang Sheng Yushou Center of Cell Biology and Immunology, School of Life Sciences and Biotechnology, Shanghai Jiao Tong University, 800 Dongchuan Road, Shanghai, 200240, People's Republic of China

Tel +86-2I-34204065

Email xuanmingyang@sjtu.edu.cn
Background: Co-stimulatory receptor agonist antibodies have shown promising antitumor efficacy in preclinical models. However, their clinical development lags due to systemic or local adverse effects of non-specific $\mathrm{T}$ cell activation. Utilization of a bispecific antibody format to reduce off-tumor immune activation is a focus of co-stimulatory receptor agonist antibody design.

Methods: In this study, a bispecific antibody with anti-CLDN18.2 and anti-CD28 moieties was produced. Its $\mathrm{T}$ cell costimulation ability was evaluated in $\mathrm{T}$ cell coculture assay in vitro. Its safety and anti-tumor efficacy were explored in mouse tumor models.

Results: Anti-CLDN18.2-anti-CD28 bispecific antibody could co-stimulate $\mathrm{T}$ cells and increase the expression of effector cytokines in a CLDN18.2-dependent manner. Treatment of anti-CLDN18.2-anti-CD28 could reduce tumor burden and increase tumor-infiltrated $\mathrm{T}$ cells. Immunosuppressive cells including tumor-associated macrophages and myeloidderived suppressor cells were also reduced without systemic adverse effects.

Conclusion: This work provided proof-of-concept evidence for a new strategy to develop a bispecific co-stimulatory activator for treating CLDN18.2 $2^{+}$tumors.

Keywords: CLDN18.2, CD28, bispecific antibody, cancer immunotherapy

\section{Introduction}

Cancer immunotherapies have made great progress in recent decades. Remarkably, various immune checkpoint antibodies and chimeric antigen receptor (CAR) T-cell therapeutics have been clinically approved. ${ }^{1-4}$ Although CAR T-cell therapy has yielded noteworthy results in treating hematologic malignancies, its efficacy for solid tumors is limited. ${ }^{4,5}$ Immune checkpoint blockade has shown broad anti-tumor efficacy across different tumor types. However, the overall objective response rate is only approximately $30 \%$, and the majority of patients are resistant to immune checkpoint blockade antibodies. ${ }^{6-8}$ Immunotherapy against solid tumors remains a challenge owing to the few specific antigen targets, high tumor heterogeneity, hostile tumor microenvironment (TME), etc. Novel therapeutics with tumor-targeted immune activation features are urgently needed for immunotherapies against solid tumors.

Appropriate tumor-specific antigens (TSAs) are the key for immunotherapy to ensure precise immune activation in tumor tissues and to avoid immune damage in normal tissues. The tight junction molecule, claudin-18.2 (CLDN18.2), is an attractive TSA for antibody-based cancer immunotherapy. CLDN18.2 in normal tissues is 
expressed exclusively in differentiated epithelial cells of the gastric mucosa, with the exception of the gastric stem cell zone. However, it is widely expressed in various tumors, including gastric, pancreatic, esophageal, ovarian, and lung tumors. ${ }^{9-11}$ All these biological characteristics have paved the way for the development of CLDN18.2-targeted therapies for solid tumors. IMAB362 (zolbetuximab) was the first monoclonal antibody developed to target CLDN18.2; its potent anti-tumor activity was proven using antibody-dependent cytotoxicity, complement-dependent cytotoxicity, induction of apoptosis, and inhibition of cell proliferation in non-clinical pancreatic ductal adenocarcinoma models. ${ }^{12,13}$ The safety and tolerance of IMAB362 were verified in clinical Phase I and II studies for the treatment of patients with metastatic gastroesophageal adenocarcinomas. ${ }^{14,15}$ Owing to the tumor specificity of CLDN18.2, other antiCLDN18.2-based targeted therapeutics such as antibodydrug conjugates and bispecific $\mathrm{T}$ cell engagers (BiTEs) have been developed. ${ }^{16,17}$ They have shown promising outcomes in pancreatic and gastric patient-derived xenograft tumor models, especially the anti-CLDN18.2/CD3 bispecific antibodies. ${ }^{18,19}$ However, CD3 bispecific antibodies can employ any $\mathrm{T}$ cell to act as an effector cell, lacking of selection on tumor antigen-specific $T$ cells. Some CD3 bispecific antibodies have been shown to induce severe cytokine release syndrome (CRS) and other side effects. ${ }^{20}$

In addition to being activators of the $\mathrm{T}$ cell receptor (TCR) signaling pathway, co-stimulation agonist antibodies have shown potent $\mathrm{T}$ cell activation ability and antitumor efficacy in preclinical models. However, the clinical development of these agonist antibodies has been hampered by undesirable off-tumor adverse effects. ${ }^{21,22} \mathrm{CD} 28$ is expressed constitutively on mouse and human $\mathrm{T}$ cells, ${ }^{23}$ and is a potential target candidate for $\mathrm{T}$ cell agonist antibodies. Unfortunately, the CD28 superagonist, TGN1412, was suspended due to severe CRS after infusion. ${ }^{24}$ It remains unclear whether tumor-targeted $\mathrm{CD} 28$ activation can maintain its $\mathrm{T}$ cell co-stimulation ability and reduce off-tumor adverse effects.

In this study, we designed an anti-CLDN18.2-antiCD28 non-Fc antibody with one arm that binds to the TSA, CLDN18.2, and the other arm that targets CD28. It showed tumor-specific $\mathrm{T}$ cell co-stimulatory activity in vitro and a good safety profile in vivo. Importantly, this bispecific anti-CLDN18.2-anti-CD28 protein exhibited potential anti-tumor efficacy mainly by increasing the number of cytotoxic $\mathrm{CD}^{+} \mathrm{T}$ cells and reshaping the immune-suppressive TME. Collectively, our data indicate that CLDN18.2-targeted CD28 activation is a potential therapeutic approach for the treatment of solid tumors, with limited adverse effects.

\section{Materials and Methods Mice}

Six- to eight-week-old female C57BL/6 mice were purchased from Beijing Vital River Laboratory Animal Technology Co., Ltd. (Beijing, China). OT-I mice were purchased from The Jackson Laboratory (Bar Harbor, ME, USA). All the mice were maintained under pathogen-free conditions and approved by the Animal Care and Use Committee of Shanghai Jiao Tong University. Animal studies were conducted in accordance with the NIH and institutional protocols and guidelines.

\section{Cell Lines and Reagents}

The Lenti-X 293 cell line was purchased from Clontech (CA, USA). B16-ovalbumin (OVA) mouse melanoma cells were provided by Hans Schreiber (University of Chicago). The usage of B16-OVA was approved by the Research Ethical Committee of Shanghai Jiao Tong University. B16-OVA-CLDN18.2 and 293-CLDN18.2 cell lines or 293-CD28 cell line were generated by infection with lentiviruses expressing mouse CLDN18.2 or mouse CD28, respectively. All the cells were cultured in DMEM (Gibco) supplemented with $10 \%$ heat-inactivated fetal bovine serum (FBS) (Gibco, Brazil), $2 \mathrm{mM}$ L-glutamine, $100 \mathrm{U} / \mathrm{mL}$ penicillin, and $100 \mu \mathrm{g} / \mathrm{mL}$ streptomycin at $37^{\circ} \mathrm{C}$ and $5 \% \mathrm{CO}_{2}$. OT-I $\mathrm{CD} 8^{+} \mathrm{T}$ cells from OT-I transgenic mice were cultured in RPMI 1640 medium supplemented similar to DMEM.

\section{Purification of Fusion Protein}

The sequence of anti-CLDN18.2 single-chain variable fragment (scFv) was derived from patent WO 2019/ $242505 \mathrm{~A} 1$, which recognizes both human and murine CLDN18.2. The anti-mouse CD28 scFv was synthesized at Genewiz (Suzhou, China) and cloned into the pCDHEF1-MCS vector. The antibodies, anti-CLDN18.2-antiCD28 and anti-CLDN18.2, were generated using Lenti-X 293 cells infected with the respective lentivirus. Proteins in the collected supernatant of cells were salted out with ammonium sulfate, dissolved, and dialyzed against PBS. 
The two proteins were purified using Ni-chelating affinity chromatography.

\section{Animal Studies}

On day 0 , wild-type $\mathrm{C} 57 \mathrm{BL} / 6$ mice at the age of $6-8$ weeks were inoculated s.c. in the right flank with $2.5 \times 10^{5}$ mouse melanoma cells (B16-OVA) overexpressing mouse CLDN18.2. For the B16-OVA-CLDN18.2 animal model, mice were peri-tumor-injected with $20 \mu \mathrm{g}$ of vehicle buffer (PBS), control protein (anti-CLDN18.2), and anti-CLDN18.2-anti-CD28 on days 8 and 11. The deletion antibody, anti-CD8 (YTS 169.4.2), was injected intraperitoneally along with anti-CLDN18.2-anti-CD28. The tumors were measured along three orthogonal axes ( $a, b$, and c) every 3 days, and the tumor volumes were calculated using the equation, $(\mathrm{a} \times \mathrm{b} \times \mathrm{c}) / 2$.

\section{Acquisition of Single Cells from Animal Tissues or Tumors}

Red blood cells from mouse spleens were lysed using Red Blood Cell Lysis buffer (Sangon Biotech, China). OT-I $\mathrm{CD}^{+} \mathrm{T}$ cells from OT-I transgenic mice were purified using the MojoSort ${ }^{\mathrm{TM}}$ Mouse CD3 $\mathrm{T}$ cell Isolation kit (BioLegend, USA). Harvested tumors were dissociated with $0.2 \mathrm{mg} / \mathrm{mL}$ DNase I (Sigma-Aldrich, USA) and $0.05 \mathrm{mg} / \mathrm{mL}$ Liberase TL (Roche, Germany) in RPMI for $25 \mathrm{~min}$ at $37^{\circ} \mathrm{C}$.

\section{Re-Stimulation of ex vivo Lymphocytes from Spleens and Lymph Nodes}

Spleens and lymph nodes of tumor-burdened mice were ground through $70 \mu \mathrm{m}$ filter strainers and washed with RPMI 1640. Red blood cell lysis was performed according to the protocol described above. Lymphocytes $\left(4 \times 10^{5}\right)$ were incubated in 96-well plates in the absence or presence of OT-I or OT-II peptide at $37{ }^{\circ} \mathrm{C}$ and $5 \% \mathrm{CO}_{2}$. IFN$\gamma$ and TNF- $\alpha$ in the supernatant were measured using a CBA kit (BD Biosciences, USA).

\section{Flow Cytometry and Analysis}

The proteins we produced were verified using monoclonal anti-His antibodies (R\&D Systems) and then stained with the secondary antibody, anti-mouse Fc-APC (Jackson ImmunoResearch). Single-cell suspensions of tumors were prepared according to the aforementioned procedure. Non-specific labeling was blocked with anti-CD16/32 (anti-FcrRII/III, clone 2.4G2) before specific labeling.
Cells were stained with anti-mouse CD45-BV605 (30F11), anti-mouse CD4-FITC (GK1.5), anti-mouse CD8 $\alpha$ APC700 (53-6.7), anti-mouse CD11c-PE (N418), antimouse MHCII-APC (M5/114.15.2), anti-mouse F4/80FITC (BM8), anti-mouse Ly6C-FITC (HK1.4), and antimouse Ly6G-PE (1A8), which were purchased from BioLegend. Anti-mouse CD69-PE (H1-2F3) and antimouse CD11b-APC700 (M1/70) were purchased from BD Biosciences and eBioscience, respectively. Anti-CD8 (YTS 169.4.2) was produced in-house. All the samples were analyzed using a Cytoflex Flow Cytometer (Beckman Coulter, Brea, CA, USA), and the data were analyzed using FlowJo software v.10.4 (TreeStar Inc., San Carlos, CA, USA).

\section{Statistics}

Statistical analysis was performed using GraphPad Prism software v.8.0. Data were expressed as means \pm standard errors of the means (SEM). The significance of the assays was determined using a two-sided Student's unpaired $t$-test. Where indicated, ${ }^{*} P<0.05,{ }^{*} P<0.01$, and $* * * P<0.001$ were considered statistically significant.

\section{Results}

\section{Generation and Characterizations of Anti-CLDN18.2-Anti-CD28 Bispecific $\mathrm{T}$ Cell Co-Stimulator}

Bispecific antibodies are considered the second generation of antibodies, and are engineered to bind to two different antigens or epitopes. As representative bispecific antibodies, BiTEs are designed to activate TCR signaling in a tumor antigen-dependent manner and have shown great success in both preclinical and clinical settings. ${ }^{25-27}$ It is unclear whether tumor-specific activation of $\mathrm{T}$ cell costimulatory signaling can boost anti-tumor activity. Hence, we designed a fusion protein, antiCLDN18.2-anti-CD28. Two scFvs recognizing murine or human CLDN18.2 and murine CD28 were fused through a flexible $(\mathrm{G} 4 \mathrm{~S})_{2}$ linker, followed by a $6 \times$ His tag for affinity purification (Figure 1A). The purified antiCLDN18.2-anti-CD28 antibody showed a molecular weight of approximately $58 \mathrm{kDa}$ based on SDS-PAGE analysis (Figure 1B).

To verify whether the two moieties of antiCLDN18.2-anti-CD28 were functional, we firstly established Lenti-X 293 and B16-OVA cell lines overexpressing murine CLDN18.2 or murine CD28. The 
A

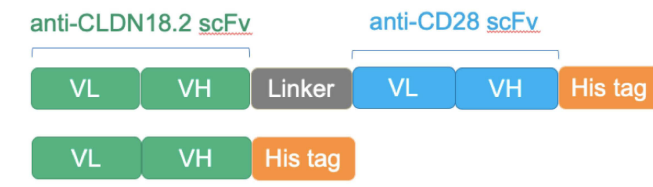

B
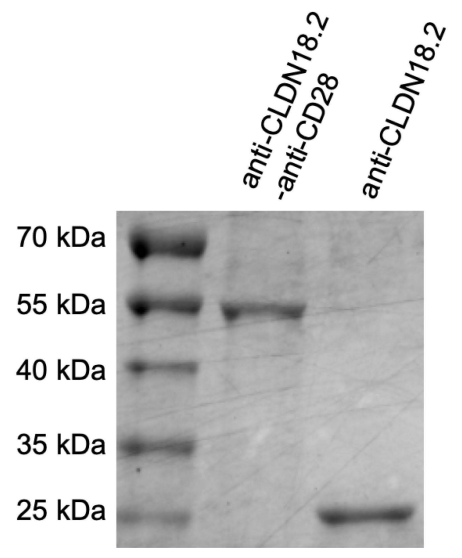

C

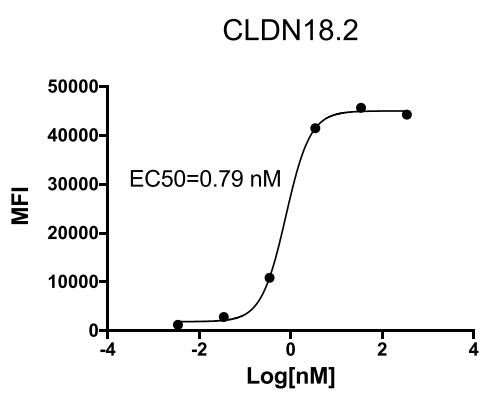

D

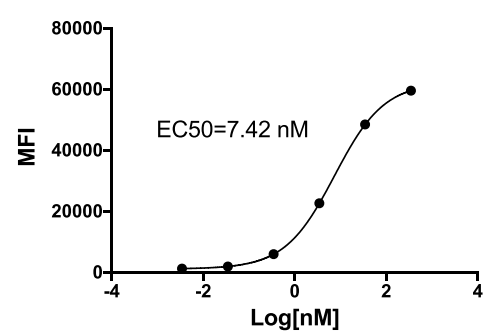

E

CD28

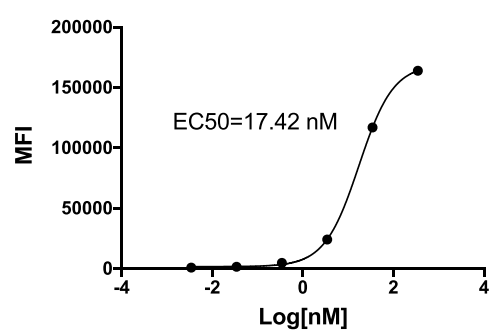

F

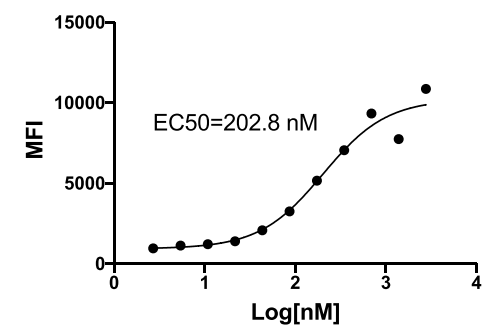

Figure I Generation and characterization of anti-CLDNI8.2-anti-CD28 bispecific antibody. (A) Schematic diagrams of bispecific antibody, anti-CLDNI8.2-anti-CD28 (top), and isotype control protein, anti-CLDNI8.2 (bottom). (B) SDS-PAGE of purified anti-CLDNI8.2-anti-CD28 (58 kDa) and anti-CLDNI8.2 (30 kDa) proteins. (C and D) Dose-dependent binding affinity of anti-CLDNI8.2 arm of the bispecific protein to Lenti-X 293-CLDNI8.2 (C) or BI6-OVA-CLDNI8.2 cells (D) expressing CLDNI8.2. (E and F) Dose-dependent binding affinity of anti-CD28 arm of the bispecific protein to Lenti-X 293-CD28 cells (E) or splenocytes from C57BL/6 mice (F).

anti-CLDN18.2-anti-CD28 fusion protein showed a strong binding ability to both 293-CLDN18.2 and B16-OVA-CLDN18.2 tumor cells, with an $\mathrm{EC}_{50}$ of 0.79 and $7.42 \mathrm{nM}$, respectively (Figure $1 \mathrm{C}$ and D). Similarly, anti-CLDN18.2-anti-CD28 could specifically bind to 293-CD28 cells with an $\mathrm{EC}_{50}$ of $17.42 \mathrm{nM}$ (Figure 1E). To test whether anti-CLDN18.2-antiCD28 could bind to endogenously expressed CD28 on $\mathrm{T}$ cells, we performed a similar binding assay with mouse splenocytes. The $\mathrm{EC}_{50}$ in splenocytes was $202.8 \mathrm{nM}$, indicating that anti-CLDN18.2-anti-CD28 had the ability to bind to endogenously expressed CD28 (Figure 1F). Thus, we successfully generated a bispecific antibody, and the functions of the two parts remained intact.

\section{Anti-CLDN18.2-Anti-CD28 Co-Stimulated T Cells in an Antigen-Dependent Manner}

CD28 is constitutively expressed on $\mathrm{T}$ cells, and provides critical co-stimulatory signals upon engaging its natural ligands, CD80 or $\mathrm{CD} 6{ }^{28}$ We tested whether anti-CLDN18.2-anti-CD28 could co-stimulate $\mathrm{T}$ cells in a CLDN18.2-dependent manner. To this end, we established CLDN18.2-negative B16-OVA and CLDN18.2-overexpressing B16-OVA-CLDN18.2 cell lines. In both cell lines, OVA was stably expressed, mimicking a TSA. The dominant peptide, OT-I, from OVA was recognized by $\mathrm{CD}^{+} \mathrm{T}$ cells from OT-I transgenic mice. ${ }^{29}$ We then co-cultured OT-I T cells, and B16-OVA and B16-OVA-CLDN18.2 cells, and tested the activation marker of $\mathrm{T}$ cells (CD69). When co-cultured with B16-OVA-CLDN18.2 cells, the expression of murine CD69 on OT-I T cells was increased by antiCLDN18.2-anti-CD28 in a dose-dependent manner (Figure 2A). However, when co-cultured with B16OVA, there was no difference in the expression of CD69 in the presence of different concentrations of anti-CLDN18.2-anti-CD28, suggesting an antigendependent activation mechanism. TNF- $\alpha$ and IFN- $\gamma$ are critical effector cytokines in activated $\mathrm{CD}^{+} \mathrm{T}$ cells. We also observed similar dose- and CLDN18.2-dependent enhancement of TNF- $\alpha$ and IFN- $\gamma$ production by antiCLDN18.2-anti-CD28 (Figure 2B and C). These data collectively suggested that anti-CLDN18.2-anti-CD28 could co-stimulate $\mathrm{T}$ cells in a CLDN18.2-dependent manner. 
A

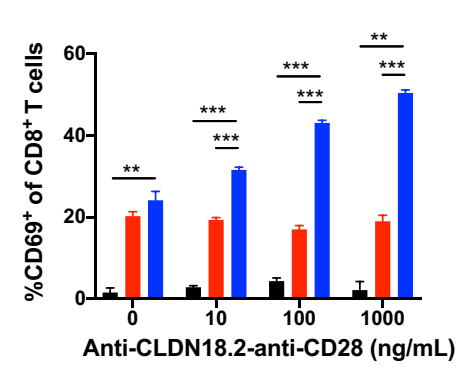

B

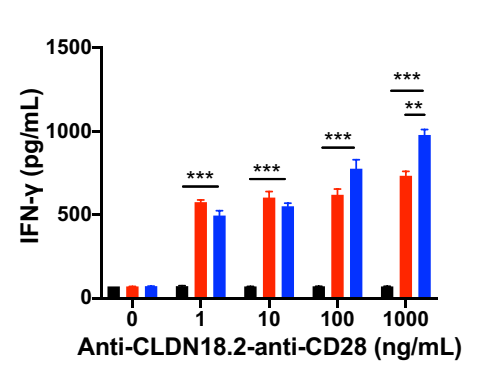

C

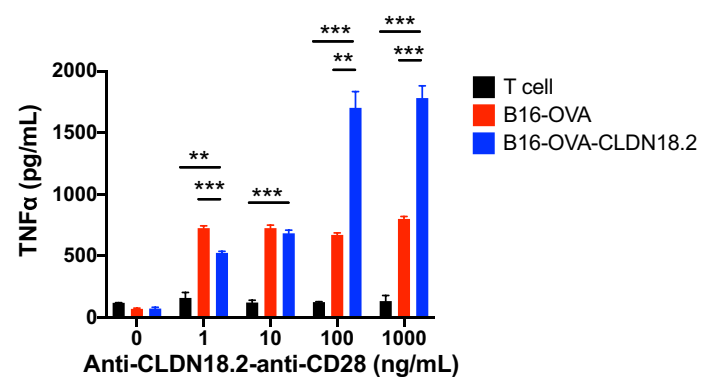

Figure 2 Co-stimulatory activation of anti-CLDNI8.2-anti-CD28 is dependent on the recognition of CLDNI8.2. $2 \times 10^{5}$ OT-I CD8 ${ }^{+}$T cells were incubated with $1 \times 10^{4}$ BI6OVA or BI6-OVA-CLDNI8.2 cells in the absence or presence of different concentrations of anti-CLDNI8.2-anti-CD28 in vitro. (A-C) Percentage of CD69 ${ }^{+}$CD $8^{+}{ }^{+}$cells after $72 \mathrm{~h}$ of incubation $(\mathbf{A})$ and release of IFN $-\gamma(\mathbf{B})$ and TNF- $\alpha(\mathbf{C})$ in the supernatant, measured after $24 \mathrm{~h}$ of incubation; $\mathrm{n}=3$, data are shown as means \pm SEM; $* * P<0.0 \mathrm{I}$, $* * * P<0.001$.

\section{Safety Profile of Anti-CLDNI8.2-Anti- CD28 Fusion Protein in Naïve Mice}

Co-stimulatory receptor agonist antibodies have been reported to induce mild to severe immune-related adverse effects in both preclinical models and clinical settings. ${ }^{24,28,29}$ To test whether our tumor antigen-specific activation of co-stimulatory receptor strategy could reduce unwanted immune activation in non-tumor tissues, non- tumor-bearing C57BL/6 mice were injected intraperitoneally with $100 \mu \mathrm{g}$ of anti-CLDN18.2-anti-CD28. Peripheral blood was collected for hematological analysis, and various tissues were collected to analyze immune cell infiltration as the evidence of immune activation. There were no obvious differences in body weight, hematological features, and infiltration of immune cells in the heart, liver, spleen, lung, kidney, and stomach (Figure 3A-E).
A

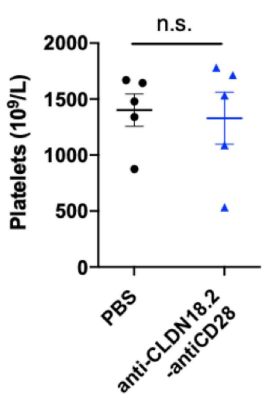

B

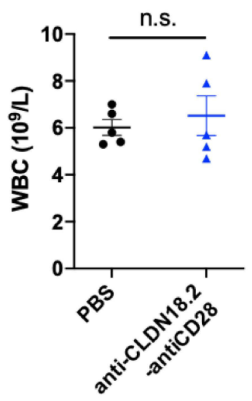

C

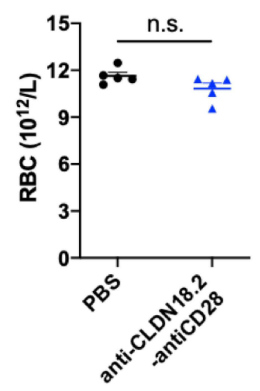

D

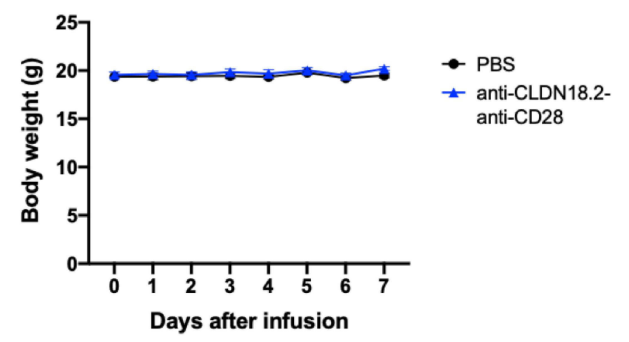

E

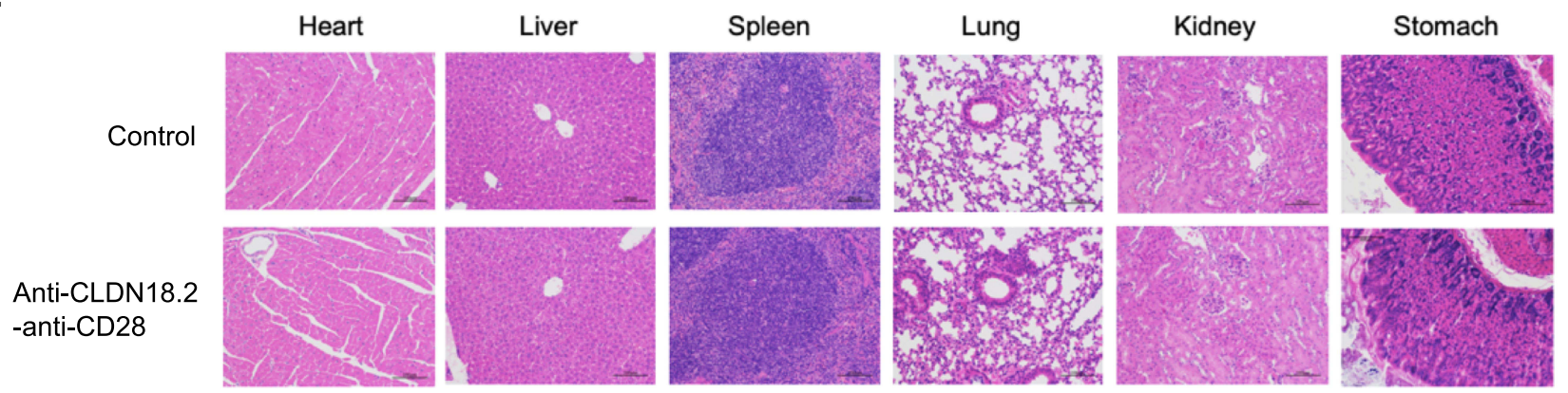

Figure 3 Safety profile of anti-CLDNI8.2-anti-CD28 in naïve C57BL/6 mice. Mice were injected i.p. with vehicle buffer (PBS) or I00 $\mu$ g anti-CLDNI8.2-anti-CD28 on day 0. (A-C) Platelet (A), WBC (B), and RBC (C) counts in the blood were measured on day 7. (D) The weights of the mice were measured every day after treatment with vehicle buffer (PBS) or anti-CLDN18.2-anti-CD28. Data are shown as means \pm SEM; $n=5$, n.s., $P>0.05$. (E) H\&E staining of major organs (heart, liver, spleen, lung, kidney, and stomach) from treated mice on day 7 . 
Although gastric epithelial cells express CLDN18.2, antiCLDN18.2-anti-CD28 treatment did not induce inflammatory immune cell infiltration in the stomach. Overall, our findings suggested that anti-CLDN18.2-anti-CD28 was well tolerated in mice, without obvious side effects.

\section{Anti-CLDN 18.2-Anti-CD28 Mainly Reduced Tumor Burden by Promoting T Cell Responses}

Owing to the enhanced ability of anti-CLDN18.2-anti-CD28 to co-stimulate $\mathrm{T}$ cells in vitro, we hypothesized that it could re-activate $\mathrm{T}$ cells in the TME by providing co-stimulation signals. To test this hypothesis, B16-OVA-CLDN18.2 tumorbearing mice were treated with PBS, anti-CLDN18.2, or antiCLDN18.2-anti-CD28. The percentage of $\mathrm{CD}^{+}$and $\mathrm{CD} 8^{+}$ T cells and the activation marker (CD69) in tumor tissues were analyzed by flow cytometry 2 days after the last treatment. Although both $\mathrm{CD}^{+}{ }^{+}$and $\mathrm{CD} 8^{+}$T cells express $\mathrm{CD} 28$, we only observed an increase in the percentage of $\mathrm{CD} 8^{+} \mathrm{T}$ cells and higher expression of $\mathrm{CD} 69$ on $\mathrm{CD}^{+} \mathrm{T}$ cells after treatment with anti-CLDN18.2-anti-CD28 (Figure 4A and B). We further tested whether anti-CLDN18.2-anti-CD28 could activate tumor-specific $\mathrm{T}$ cell responses. Splenocytes from treated mice were re-stimulated with OT-I or OT-II peptide, which mimics the TSA. The splenocytes produced more IFN- $\gamma$ and TNF- $\alpha$ in the anti-CLDN18.2-anti-CD28-treated group, indicating elevated tumor-specific $\mathrm{CD}^{+}$and $\mathrm{CD}^{+} \mathrm{T}$ cell responses (Figure $4 \mathrm{C}$ and $\mathrm{D}$ ). Consistent with the increase in the number of $\mathrm{T}$ cells and the activation status, antiCLDN18.2-anti-CD28 reduced tumor burden in vivo compared with that in the PBS- and anti-CLDN18.2-treated groups (Figure 4E). There was no difference in body weight among the groups (Figure 4F). Moreover, we used the deletion antibody to verify the necessity of $\mathrm{CD}^{+} \mathrm{T}$ cells for antiCLDN18.2-anti-CD28 antibody efficacy in B16-OVACLDN18.2 mouse models. As expected, the presence of $\mathrm{CD}^{+} \mathrm{T}$ cells affected the function of anti-CLDN18.2-antiCD28 (Figure 4G and H). Therefore, the anti-CLDN18.2 -anti-CD28 bispecific antibody could provide co-stimulation signals in the TME to promote anti-tumor $\mathrm{T}$ cell responses, which subsequently reduced the tumor burden.

\section{Anti-CLDN18.2-Anti-CD28 Reduced the Immunosuppressive Components in} Tumor Microenvironment

Cancer responding to immunotherapy does not directly depend only on tumor infiltration by cytotoxic $\mathrm{CD} 8^{+}$
$\mathrm{T}$ cells; other tumor-associated immune cells, such as tumor-associated macrophages (TAMs) and myeloidderived suppressor cells (MDSCs), also play a protective or opposite role in the process of tumor killing. To test whether anti-CLDN18.2-anti-CD28 reshaped the immunosuppressive TME, we analyzed other types of immune cells in TME on days 4 and 10 after treatment with antiCLDN18.2-anti-CD28. Decreased TAMs of intra-tumoral infiltration were found in mice treated with antiCLDN18.2-anti-CD28 (Figure 5A). TAMs in tumors could release cytokines and chemokines that suppress anti-tumor immunity, indicating infusion of antiCLDN18.2-anti-CD28 might affect the suppressive microenvironment. ${ }^{30,31}$ The number of MDSCs, another important immunosuppressive myeloid-derived population, including $\mathrm{Ly}_{6} \mathrm{C}^{+} \mathrm{CD} 11 \mathrm{~b}^{+}$(mMDSCs) and $\mathrm{Ly}_{6} \mathrm{G}^{+} \mathrm{CD} 11 \mathrm{~b}^{+}$(gMDSCs) of mice treated with antiCLDN18.2-anti-CD28 showed a trend of reduction (Figure 5B and C) on day 4 after treatment. Previous studies have reported that early infiltration of MDSCs within primary tumors was low and it would gradually increase in the late stage. ${ }^{32,33}$ Hence, we investigated the MDSCs again after several days. Treatment of antiCLDN18.2-anti-CD28 reduced both types of MDSCs in TME (Figure 5D and E). Taken together, antiCLDN18.2-anti-CD28 could also influence the growth of tumor by improving the suppressive TME, suggesting the validity of antigen CLDN18.2 and potent activation capability of CD28 in tumor killing.

\section{Discussion}

In this study, we designed a CLDN18.2-targeted bispecific antibody by connecting two scFvs against CLDN18.2 and CD28. It co-stimulated $\mathrm{T}$ cells in a TSA-specific manner in vitro, increased $\mathrm{T}$ cell infiltration and activation in vivo, and showed a promising anti-tumor effect in mouse tumor models.

The initial idea for a bispecific $T$ cell activation antibody was proposed by linking a TSA with CD3 to one molecule; this antibody showed potent anti-tumor efficacy. ${ }^{18}$ However, this strategy transiently activates all $\mathrm{T}$ cells without antigen specificity. The risk of excessive stimulation in a cytokine storm cannot be ignored. ${ }^{24}$ CD28, due to its co-stimulatory nature, is activated upon TCR signal activation, showing a tumor antigen-specific $\mathrm{T}$ cell activation. A recent study described a type of bispecific antibody that combined another TSA and CD28, which promoted long-term anti-tumor immunity, 
A

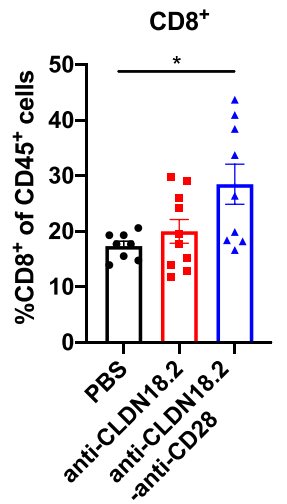

B

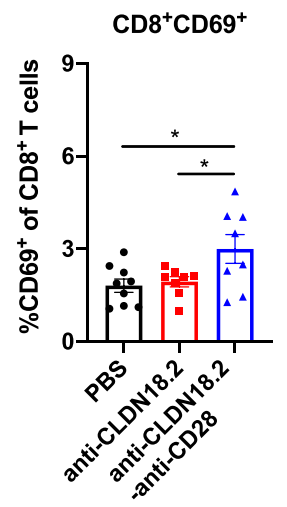

$\mathrm{CD}^{+} \mathrm{CD}^{+} 9^{+}$

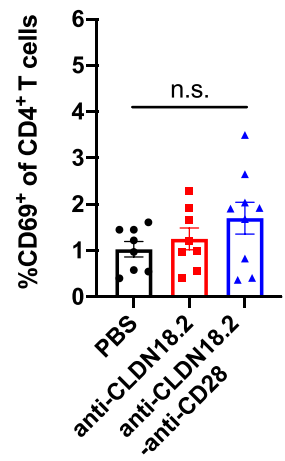

C
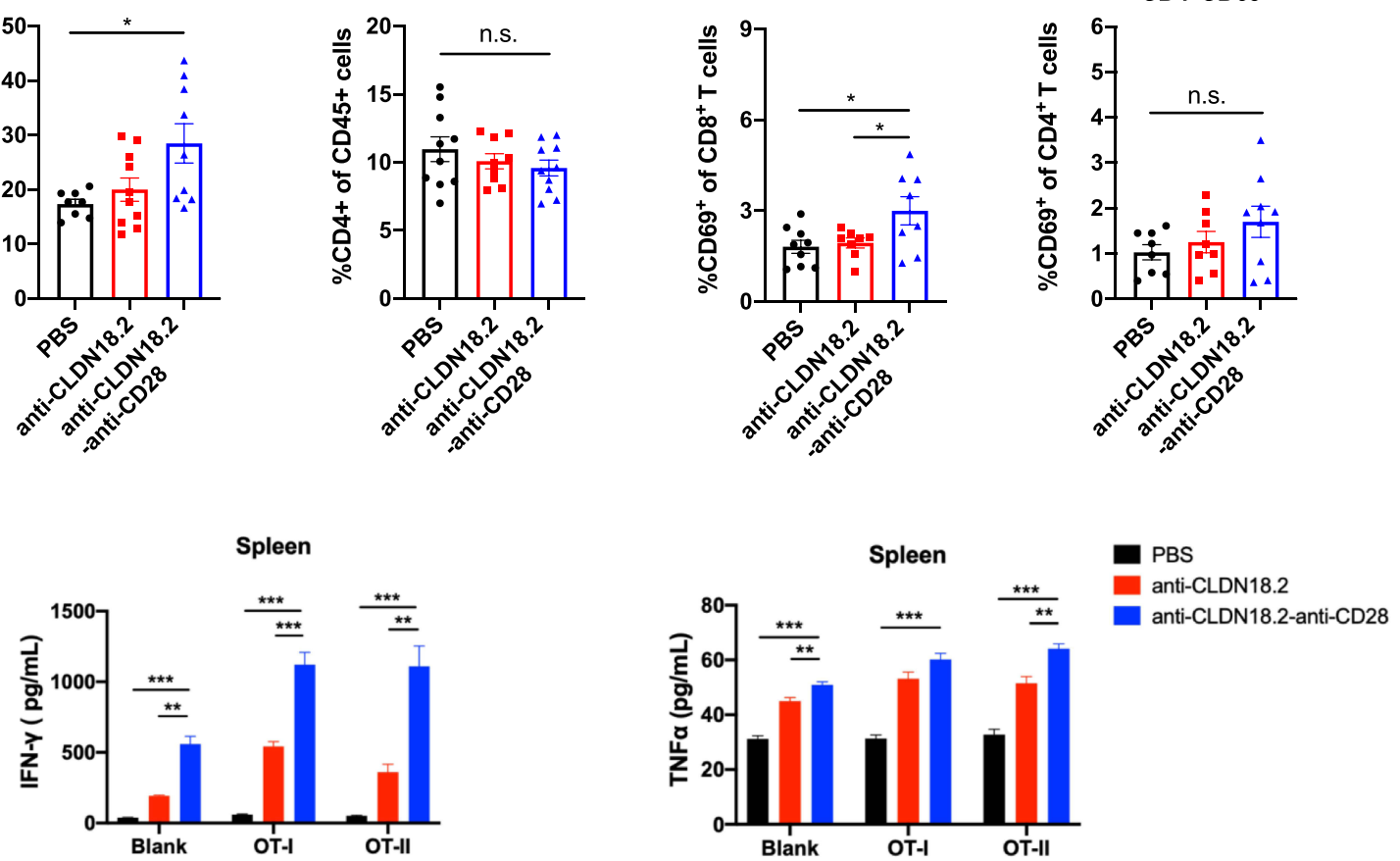

D

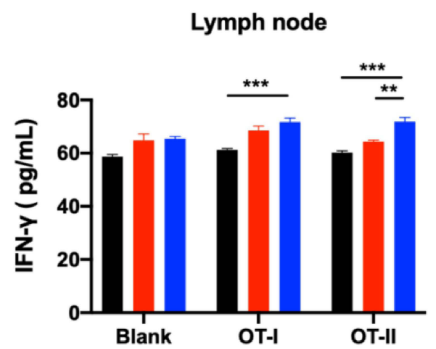

E

- PBS

- anti-CLDN18.2

$\star$ anti-CLDN18.2-anti-CD28

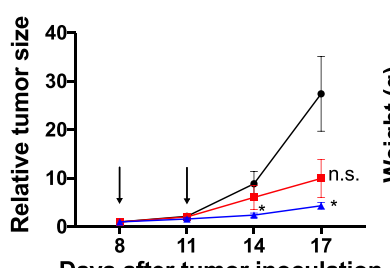

Days after tumor inoculation
$\mathbf{F}$

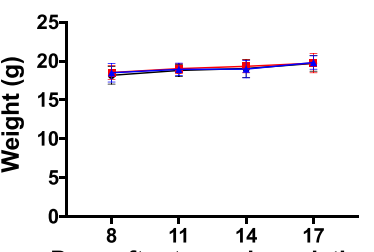

Days after tumor inoculation

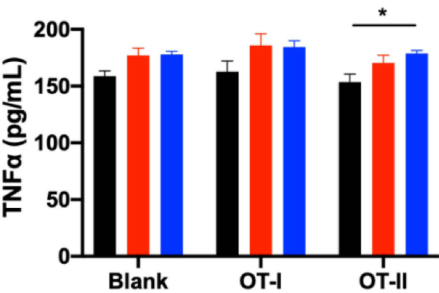

G

H

- PBS

- anti-CLDN18.2-anti-CD28

- anti-CLDN18.2-anti-CD28 + anti-CD8

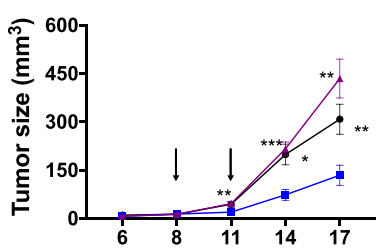

Days after tumor inoculation

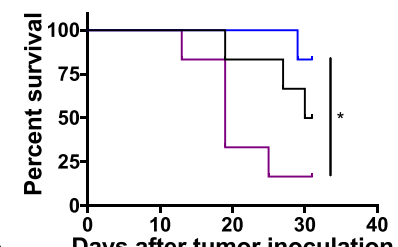

Days after tumor inoculation

Figure 4 Anti-CLDN18.2-anti-CD28 mainly reduced the tumor burden by activating long-term tumor-specific T cells in TME. (A-D) C57BL/6 mice were inoculated subcutaneously with $2.5 \times 10^{5}$ BI6-OVA-CLDNI8.2 cells on day 0. Vehicle buffer (PBS), anti-CLDNI8.2, and bispecific antibody (anti-CLDNI8.2-anti-CD28) were peritumor-injected on days 8 and II. Mice were euthanized on day 13. Percentage of CD8 ${ }^{+}$or $\mathrm{CD}^{+} \mathrm{T}$ cells $(\mathbf{A})$ in tumor-infiltrating myeloid populations and expression of CD69 on $\mathrm{CD}^{+}$or $\mathrm{CD}^{+} \mathrm{T}$ cells (B) in tumor-infiltrating lymphocytes were analyzed on day 13. (C and D) Splenic and lymph node cells from mice were separated and incubated in the absence or presence of OT-I or OT-II peptide. IFN- $\gamma$ and TNF- $\alpha$ in the supernatants of splenocyte (C) and lymph node cell (D) cultures were measured after $48 \mathrm{~h} ; \mathrm{n}=5$. ( $\mathbf{E}$ and $\mathbf{F}) \mathrm{C} 57 \mathrm{BL} / 6$ mice were inoculated subcutaneously with $2.5 \times 10^{5}$ BI6-OVA-CLDNI8.2 tumor cells. Tumor size (E) and weight (F) of mice. Black arrows indicate the time points of antibody injection; $n=5-6$. ( $\mathbf{G}$ and $\mathbf{H}$ ) Mice inoculated subcutaneously with BI6-OVA-CLDNI8.2 were treated with vehicle buffer and antiCLDN18.2-anti-CD28 with or without deletion antibody (anti-CD8) on days 8 and II. The tumor size $(\mathbf{G})$ and survival curves $(\mathbf{H})$ were measured and recorded, respectively. Black arrows indicate the time points of antibody injection; $n=6$. Statistical significance of survival experiments was determined using the Mantel-Cox test. All the data are shown as means \pm SEM; $* P<0.05$, **P $<0.01$, ***P $<0.001$. 
A

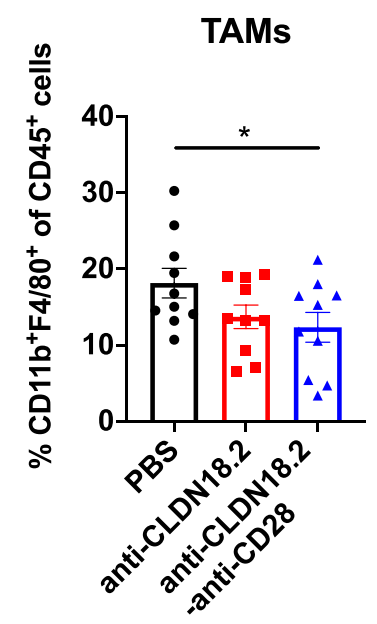

B

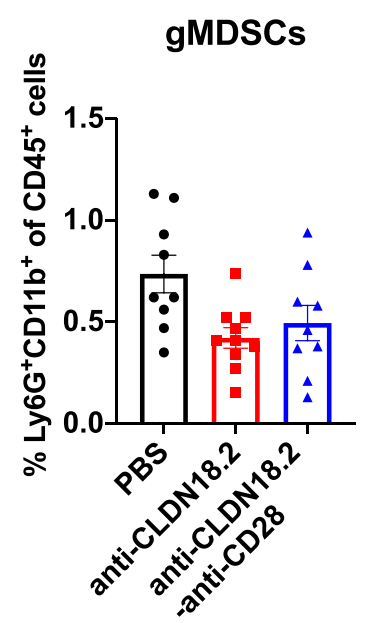

C

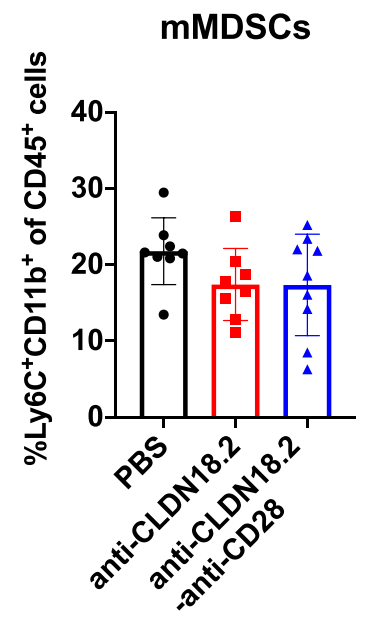

D

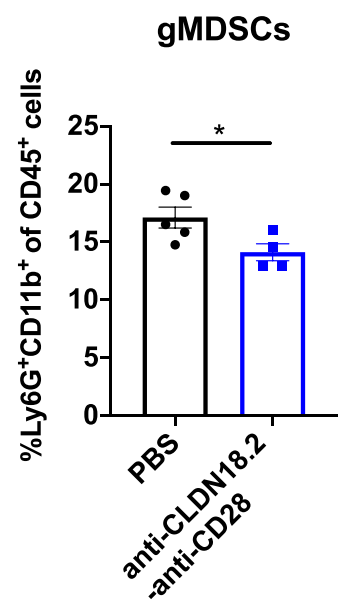

$\mathrm{E}$

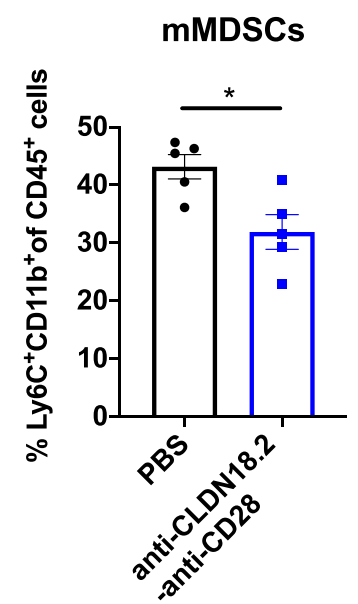

Figure 5 Anti-CLDN18.2-anti-CD28 reduced the immunosuppressive components in tumor microenvironment. Tumor-infiltrating immune cells were acquired from BI6OVA-CLDNI8.2 mouse models. (A) Proportion of intra-tumoral macrophage subpopulation from C57BL/6 mice. (B and C) Proportion of intra-tumoral CDI Ib ${ }^{+}$Ly6G ${ }^{+}$ MDSCs (B) and CDI Ib ${ }^{+}$Ly6C ${ }^{+}$MDSCs (C) from C57BL/6 mice. In (A-C), tumors were resected and analyzed on day 12 after BI6-OVA-CLDNI8.2 inoculation; $\mathrm{n}=8-10$,

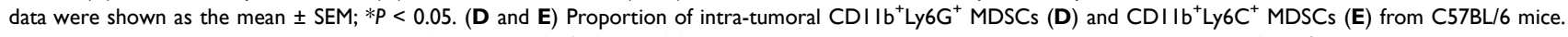
Tumors were resected and analyzed on day 18 after BI6-OVA-CLDNI8.2 tumor cells inoculation; $n=5$, data were shown as means \pm SEM; $* P<0.05$.

suggesting the potential of CD28 in the development of bispecific antibodies. ${ }^{16}$

CD28 is a co-stimulatory receptor of $\mathrm{T}$ cells that augments and sustains $\mathrm{T}$ cell activation and growth. Since CD28 is constitutively expressed on T cells, we believe that CD28, which targets $\mathrm{T}$ cell activation, has a broader application potential than other transiently expressed co-stimulatory receptors. We observed enhanced activity of most intra-tumoral $\mathrm{CD}^{+} \mathrm{T}$ cells of mice treated with anti-CLDN18.2-anti-CD28 in B16CLDN18.2 mouse models, in accordance with the in vitro experimental results. Besides, the immunosuppressive cells including TAMs and MDSCs also showed a trend of reduction. It is possible that the treatment of anti-CLDN18.2-anti-CD28 reduced the tumor burden through enhanced anti-tumor $\mathrm{T}$ cell response, which indirectly suppressed MDSCs proliferation by limiting tumor-derived MDSC-promoting factors. ${ }^{34}$ Since CD28agonistic antibodies induce severe side effects in clinical settings, we also evaluated the safety profile after antiCLDN18.2-anti-CD28 infusion. We did not observe obvious side effects after anti-CLDN18.2-anti-CD28 treatment, even in CLDN18.2-expressing gastric tissues. These data collectively suggested that antiCLDN18.2-anti-CD28 was both effective and safe in mouse tumor models. 
The data on the curve of tumor growth in vivo indicated that the control protein, anti-CLDN18.2, also exhibited anti-tumor efficacy to some extent; however, its specific mechanism was unclear. To test whether the antiCLDN18.2 scFv could directly inhibit tumor growth in vitro, we incubated B16-OVA or B16-OVACLDN18.2 cells with different concentrations of the scFv and found that it had no effect on the growth of both tumor cells (Figure S1). It is possible that anti-CLDN18.2 blocks the interaction of CLDN18.2 with other components from the TME, thereby indirectly affecting tumor cells or the tumor-promoting TME.

Although checkpoint inhibition with monoclonal antibodies such as anti-PD-1/PD-L1 has been widely used to treat solid tumors in clinical settings, the objective response rate is approximately $20-30 \%{ }^{35}$ Low tumor mutation burdens, less tumor infiltration, and low expression of PD-1/PD-L1 may contribute to the unresponsiveness to PD-1/PD-L1 antibodies. Instead of blocking the inhibitory mechanism, our antibody focused on activating $\mathrm{T}$ cells, independent of the inhibitory mechanism; this is a potential strategy to treat patients unresponsive to PD-1/ PD-L1 antibodies. A recent study described a type of antibody that merged TSAs and anti-CD28 into one molecule; it synergized with anti-PD-1 and enhanced the overall efficacy of the immunotherapy. ${ }^{36}$ Therefore, the combination of anti-CLDN18.2-anti-CD28 and anti-PD-1 can be considered for further exploration.

The limitations of this study include the following. Owing to the lack of an Fc domain, our antiCLDN18.2-anti-CD28 fusion protein has limited durability and stability in vivo. We performed peri-tumor injection to overcome these limitations in proof-of-concept experiments. Due to the lack of a CLDN18.2-expressing mouse tumor cell line, we used ectopic CLDN18.2-expressing melanoma B16-OVA cells and pancreatic tumor Pan02 cells in our tumor models. However, antiCLDN18.2-anti-CD28 showed only modest anti-tumor efficacy in the Pan02-CLDN18.2 mouse model (data not shown). The endogenous expression of CLDN18.2 probably varies in different tumor cells, and it is important to investigate the influence of different CLDN18.2 densities on the anti-CLDN18.2-anti-CD28-induced T cell activation capability.

In summary, bispecific antibodies are useful for generating site-specific immune responses. Our CLDN18.2-targeted CD28 activation bispecific antibody shows tumor-specific $\mathrm{T}$ cell co-stimulatory activity in vitro and potent anti-tumor efficacy in mouse models, with limited adverse effects, providing support for extensive exploration of CD28 for the treatment of solid tumors in clinical settings.

\section{Institutional Review Board Statement}

All the animal studies were approved by the Animal Care and Use Committee of Shanghai Jiao Tong University (ethics code: A2017043, approved on July 18, 2017).

\section{Acknowledgments}

We thank Dr. Hans Schreiber (University of Chicago) for providing B16-OVA mouse melanoma cells.

\section{Author Contributions}

All authors made substantial contributions to conception and design, acquisition of data, or analysis and interpretation of data; took part in drafting the article or revising it critically for important intellectual content; agreed to submit to the current journal; gave final approval of the version to be published; and agree to be accountable for all aspects of the work.

\section{Funding}

Xuanming Yang was supported by the National Natural Science Foundation of China (81971467) and Shanghai Jiao Tong University Scientific and Technological Innovation Funds (2019QYA11).

\section{Disclosure}

The authors declare that the research was conducted in the absence of any commercial or financial relationships that could be construed as potential conflicts of interest.

\section{References}

1. Maury S, Chevret S, Thomas X, et al. Addition of rituximab improves the outcome of adult patients with CD20-positive, Ph-negative, B-cell precursor acute lymphoblastic leukemia (BCP-ALL): results of the randomized Graall-R 2005 study. Blood. 2015;126(23):1. doi:10.1182/blood.V126.23.1.1

2. Jabbour E, O'Brien S, Ravandi F, Kantarjian H. Monoclonal antibodies in acute lymphoblastic leukemia. Blood. 2015;125 (26):4010-4016. doi:10.1182/blood-2014-08-596403

3. Ansell SM, Lesokhin AM, Borrello I, et al. PD-1 blockade with nivolumab in relapsed or refractory Hodgkin's lymphoma. $N$ Engl J Med. 2015;372(4):311-319. doi:10.1056/NEJMoa1411087

4. Kosti P, Maher J, Arnold JN. Perspectives on chimeric antigen receptor T-cell immunotherapy for solid tumors. Front Immunol. 2018;9:1104. doi:10.3389/fimmu.2018.01104

5. Newick K, O'Brien S, Moon E, Albelda SM. CAR T cell therapy for solid tumors. Annu Rev Med. 2017;68:139-152. doi:10.1146/annurevmed-062315-120245 
6. Weber JS, D'Angelo SP, Minor D, et al. Nivolumab versus chemotherapy in patients with advanced melanoma who progressed after anti-CTLA-4 treatment (CheckMate 037): a randomised, controlled, open-label, Phase 3 trial. Lancet Oncol. 2015;16(4):375-384. doi:10.1016/S1470-2045(15)70076-8

7. Schadendorf D, Hodi FS, Robert C, et al. Pooled analysis of long-term survival data from phase II and phase III trials of ipilimumab in unresectable or metastatic melanoma. J Clin Oncol. 2015;33 (17):1889-1894. doi:10.1200/JCO.2014.56.2736

8. Fuchs CS, Ozguroglu M, Bang YJ, et al. Pembrolizumab versus paclitaxel for previously treated patients with PD-L1-positive advanced gastric or gastroesophageal junction cancer (GC): update from the phase III KEYNOTE-061 trial. J Clin Oncol. 2020;38 (15):4503.

9. Sahin U, Koslowski M, Dhaene K, et al. Claudin-18 splice variant 2 is a pan-cancer target suitable for therapeutic antibody development. Clin Cancer Res. 2008;14:7624-7634. doi:10.1158/1078-0432.CCR08-1547

10. Tanaka M, Shibahara J, Fukushima N, et al. Claudin-18 is an early-stage marker of pancreatic carcinogenesis. J Histochem Cytochem. 2011;59(10):942-952. doi:10.1369/0022155411420569

11. Soini Y, Takasawa A, Eskelinen M, et al. Expression of claudins 7 and 18 in pancreatic ductal adenocarcinoma: association with features of differentiation. J Clin Pathol. 2012;65(5):431-436. doi:10.1136/ jclinpath-2011-200400

12. Woll S, Schlitter AM, Dhaene K, et al. Claudin 18.2 is a target for IMAB362 antibody in pancreatic neoplasms. Int J Cancer. 2014;134 (3):731-739. doi:10.1002/ijc. 28400

13. Tureci O, Mitnacht-Kraus R, Woll S, Yamada T, Sahin U. Characterization of zolbetuximab in pancreatic cancer models. Oncoimmunology. 2019;8(1):e1523096. doi:10.1080/ 2162402x.2018.1523096

14. Schuler MH, Zvirbule Z, Lordick F, et al. Safety, tolerability, and efficacy of the first-in-class antibody IMAB362 targeting claudin 18.2 in patients with metastatic gastroesophageal adenocarcinomas. J Clin Oncol. 2013;31(15):4080. doi:10.1200/jco.2013.31.15_suppl.4080

15. Sahin U, Schuler M, Richly H, et al. A phase I dose-escalation study of IMAB362 (Zolbetuximab) in patients with advanced gastric and gastro-oesophageal junction cancer. Eur J Cancer. 2018;100:17-26. doi:10.1016/j.ejca.2018.05.007

16. Zhu G, Foletti D, Liu X, et al. Targeting CLDN18.2 by CD3 bispecific and ADC modalities for the treatments of gastric and pancreatic cancer. Sci Rep. 2019;9(1):8420. doi:10.1038/s41598-019-44874-0

17. Chasov V, Zaripov M, Mirgayazova R, et al. Promising new tools for targeting p53 mutant cancers: humoral and cell-based immunotherapies. Front Immunol. 2021;12:3223. doi:10.3389/ fimmu.2021.707734

18. Perez P, Hoffman RW, Shaw S, Bluestone JA, Segal DM. Specific targeting of cytotoxic $\mathrm{T}$ cells by anti-T3 linked to anti-target cell antibody. Nature. 1985;316(6026):354-356. doi:10.1038/316354a0

19. Hoffman L, Gore L. Blinatumomab, a bi-specific anti-CD19/CD3 BiTE $^{\circledR}$ antibody for the treatment of acute lymphoblastic leukemia: perspectives and current pediatric applications. Front Oncol. 2014;4:63. doi: $10.3389 /$ fonc. 2014.00063

20. Wu Z, Cheung NV. T cell engaging bispecific antibody (T-BsAb): from technology to therapeutics. Pharmacol Ther. 2018;182:161-175. doi:10.1016/j.pharmthera.2017.08.005

21. Vonderheide RH, Glennie MJ. Agonistic CD40 antibodies and cancer therapy. Clin Cancer Res. 2013;19(5):1035-1043. doi:10.1158/10780432.CCR-12-2064
22. Qi X, Li F, Wu Y, et al. Optimization of 4-1BB antibody for cancer immunotherapy by balancing agonistic strength with Fc $\gamma \mathrm{R}$ affinity. Nat Commun. 2019;10(1):2141. doi:10.1038/s41467-019-10088-1

23. Correnti CE, Laszlo GS, de van der Schueren WJ, et al. Simultaneous multiple interaction T-cell engaging (SMITE) bispecific antibodies overcome bispecific T-cell engager (BiTE) resistance via CD28 co-stimulation. Leukemia. 2018;32(5):1239-1243. doi:10.1038/ s41375-018-0014-3

24. Suntharalingam G, Perry MR, Ward S, et al. Cytokine storm in a Phase 1 trial of the anti-CD28 monoclonal antibody TGN1412. $N$ Engl J Med. 2006;355(10):1018-1028. doi:10.1056/ NEJMoa063842

25. Jabbour EJ, Gokbuget N, Kantarjian HM, et al. Transplantation in adults with relapsed/refractory acute lymphoblastic leukemia who are treated with blinatumomab from a phase 3 study. Cancer. 2019;125 (23):4181-4192. doi:10.1002/cncr.32335

26. Dombret H, Topp MS, Schuh AC, et al. Blinatumomab versus chemotherapy in first salvage or in later salvage for B-cell precursor acute lymphoblastic leukemia. Leuk Lymphoma. 2019;60 (9):2214-2222. doi:10.1080/10428194.2019.1576872

27. Loffler A, Kufer P, Lutterbuse R, et al. A recombinant bispecific single-chain antibody, CD19 x CD3, induces rapid and high lymphoma-directed cytotoxicity by unstimulated $\mathrm{T}$ lymphocytes. Blood. 2000;95(6):2098-2103. doi:10.1182/blood.V95.6.2098

28. Vonderheide RH, Flaherty KT, Khalil M, et al. Clinical activity and immune modulation in cancer patients treated with CP-870,893, a novel CD40 agonist monoclonal antibody. J Clin Oncol. 2007;25 (7):876-883. doi:10.1200/Jco.2006.08.3311

29. Dubrot J, Milheiro F, Alfaro C, et al. Treatment with anti-CD137 mAbs causes intense accumulations of liver $\mathrm{T}$ cells without selective antitumor immunotherapeutic effects in this organ. Cancer Immunol Immun. 2010;59(8):1223-1233. doi:10.1007/s00262-010-0846-9

30. Pollard JW. Tumour-educated macrophages promote tumour progression and metastasis. Nat Rev Cancer. 2004;4(1):71-78. doi:10.1038/ nrc 1256

31. Kitamura T, Qian BZ, Pollard JW. Immune cell promotion of metastasis. Nat Rev Immunol. 2015;15(2):73-86. doi:10.1038/ nri3789

32. Jordan KR, Amaria RN, Ramirez O, et al. Myeloid-derived suppressor cells are associated with disease progression and decreased overall survival in advanced-stage melanoma patients. Cancer Immunol Immunother. 2013;62(11):1711-1722. doi:10.1007/s00262-013-1475-X

33. Kumar V, Patel S, Tcyganov E, Gabrilovich DI. The nature of myeloid-derived suppressor cells in the tumor microenvironment. Trends Immunol. 2016;37(3):208-220. doi:10.1016/j.it.2016.01.004

34. Groth $\mathrm{C}, \mathrm{Hu} \mathrm{X}$, Weber $\mathrm{R}$, et al. Immunosuppression mediated by myeloid-derived suppressor cells (MDSCs) during tumour progression. Br J Cancer. 2019;120(1):16-25. doi:10.1038/s41416018-0333-1

35. Hodi FS, O’Day SJ, McDermott DF, et al. Improved survival with ipilimumab in patients with metastatic melanoma. New Engl J Med. 2010;363(8):711-723. doi:10.1056/NEJMoa1003466

36. Waite JC, Wang B, Haber L, et al. Tumor-targeted CD28 bispecific antibodies enhance the antitumor efficacy of PD-1 immunotherapy. Sci Transl Med. 2020;12(549):eaba2325. doi:10.1126/scitranslmed. aba2325 


\section{Publish your work in this journal}

Cancer Management and Research is an international, peer-reviewed open access journal focusing on cancer research and the optimal use of preventative and integrated treatment interventions to achieve improved outcomes, enhanced survival and quality of life for the cancer patient.

The manuscript management system is completely online and includes a very quick and fair peer-review system, which is all easy to use. Visit http://www.dovepress.com/testimonials.php to read real quotes from published authors. 